Preprint version 11th Int. Conf. on Swarm Intelligence, ANTS 2018, Rome Italy (2018)

\title{
A Study on Force-based Collaboration in Flying Swarms
}

\author{
Chiara Gabellieri ${ }^{2}[0000-0002-0051-2941]$, Marco Tognon ${ }^{10000-0003-1700-9637],}$ \\ Lucia Pallottino $^{2[0000-0002-9480-8857]}$, and Antonio Franchi ${ }^{10000-0002-5670-1282]}$ \\ 1 LAAS-CNRS, Université de Toulouse, CNRS, Toulouse, France, \\ marco.tognon@laas.fr, antonio.franchi@laas.fr \\ 2 Centro di Ricerca E. Piaggio, Dipartimento di Ingegneria dell'Informazione, Università di \\ Pisa, Italy lucia.pallottinodunipi.it
}

\begin{abstract}
This work investigates collaborative aerial transportation by swarms of agents based only on implicit information, enabled by the physical interaction among the agents and the environment. Such a coordinating mechanism in collaborative transportation is a basic skill in groups of social animals. We consider cable-suspended objects transported by a swarm of flying robots and we formulate several hypothesis on the behavior of the overall system which are validated thorough numerical study. In particular, we show that a nonzero internal force reduces to one the number of asymptotically stable equilibria and that the internal force intensity is directly connected to the convergence rate. As such, the internal force represents the cornerstone of a communication-less cooperative manipulation paradigm in swarms of flying robots. We also show how a swarm can achieve a stable transportation despite the imprecise knowledge of the system parameters.
\end{abstract}

\section{Introduction}

Cooperative transportation without explicit communication, but based only on on the indirect exchange of information through the physical interaction with the environment is a very important feature for social animals. From a scientific point of view, the problem has been addressed as Stigmery theory [8]; later, it has been regarded as the main coordinating mechanism in groups of ants for object transportation [9] (see Fig. 1 on the left) and has been indeed observed and studied in $[3,5,6,13]$.

Such skills observed in nature have inspired researchers to transfer them to swarms of simple robotic agents. In fact, avoiding explicit communication would reduce hardware and software complexity, and overcome possible communication failure issues. So far, the interest has been mainly focused on terrestrial systems [3, 9, 17, 22], where the possibility of decentralized transportation based on physical interactions has been proved. Instead, in this work, we are interested in the communication-less aerial transportation of objects by swarms. This is particularly interesting not only from a scientific point of view, thanks to the higher complexity of the problem, but also because a simple, robust and scalable object aerial manipulation technique could meet the requirements demanded by many real applications. Aerial transportation can benefit from 

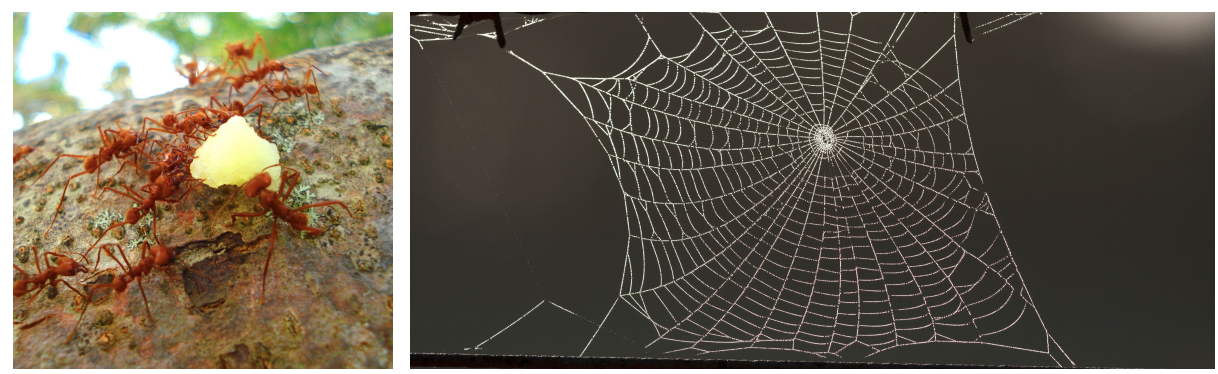

Fig. 1: Left: red ants in cooperative transportation. Right: the tension in spider webs influences their natural frequency [2].

a larger workspace and the independence from uneven terrains. However, aerial robots, e.g., multi-rotors, though agile and low cost, are typically characterized by a limited payload. Hence, a cooperative approach is a very suitable solution. Some examples of applications can be found in industrial contexts, in agriculture, and in search and rescue missions to carry necessary equipment or first aid.

Considering the lack of results for aerial systems compared to grounded ones, our purpose is to start filling this gap. In particular, we investigate the possibility of a communication-less approach for cooperative aerial manipulation with a swarm of flying agents. Furthermore, we investigate if and how the load and the cable physically connecting the robots may play the role of an implicit communication channel exploiting the forces exchanged. To the best of our knowledge, this is the first work proposing a bio-inspired algorithm for communication-less aerial manipulation by flying swarms, going beyond the two-robot- 'only' scenario, recently considered in [7,20,21].

The algorithm that we propose exploits a leader-follower paradigm where the leader agent knows where to go and hence steers the object toward the desired position. On the other hand, the follower agents follow the leader and help to sustain the weight and manipulate the load, exploiting only the implicit information contained in the force received from the load itself. It has been observed that also groups of army ants Eciton burchelli [5] and Dorylus wilverthi [6] adopt a distinct caste distribution in transportation groups, in the sense that groups have a significant tendency to contain only one submajor, i.e., a particular type of ant. These species of army ants have proved to be very efficient in transporting objects together. Additionally, in the same works, it has been noticed that it is usually a single ant, the submajor, that starts the motion of the object, and then the rest of the group moves accordingly. Such a behavior is actually replicated in the leader-follower paradigm proposed in this paper.

While animals usually deploy items by directly touching them (direct manipulation), in our framework we have chosen an indirect manipulation technique of the load through cables. This choice is motivated by different reasons and it has been proved to be a very effective solution for cooperative aerial transportation [10-12, 19,23]. First of all, a cable attached to the agent center of mass allows to minimize the coupling between the rotational dynamics of the agent itself from the rest of the system dynamics. This is particularly useful for aerial agents that are underactuated - the most common 
case - since they need to change their attitude to be able to apply forces in any direction. Furthermore, compared to other possible decoupling gripping mechanisms, as the ones in [14], cables are simpler, low cost, and in general lighter. In this paper we demonstrate through several numerical simulations that a swarm of $N$ flying agents is capable of collaborative manipulation skills based only on implicit communication. We show that a twofold major role is played by the internal force applied to the transported object. Internal forces are forces applied at the contact points on the object that stretch or compress it without producing any movement, since they counterbalance each other. The condition of zero internal force corresponds to the case in which the agents transport the object while keeping the cables vertical and applying only a force that compensates for the gravity. Firstly, we have found that nonzero internal forces allow the swarm to univocally set the attitude of the commonly transported object, and secondly, that larger internal forces reduce the convergence time of the overall system to such unique equilibrium. This creates an interesting analogy with the role of tension in spider webs, see Fig. 1-right. The breadth of analysis covers also the thorough investigation of the leader forces depending on the swarm parameters and the analysis of the benefits of a saturated nonlinear law for the leader force in order to tradeoff compliance/safety and transportation accuracy.

The paper structure is the following: Sec. 2 illustrates the dynamic model of the system. Then, we formulate the hypotheses regarding the properties of the swarm, supported by the numerical results presented in Sec. 3. A thorough discussion follows in Sec. 4. Final conclusions and future developments are presented in Sec 5.

\section{Model}

The system is composed by a set of $N$ flying agents attached to a cable suspended load that must be deployed to a particular configuration. In our framework, each agent interacts with the environment, hence we aim at a soft response similar to the behavior of human or of animal during everyday interaction tasks. We model the commonly transported object (the load) as a rigid body. The attitude of the load is parametrized by Euler angles yaw, pitch and roll, indicated with $\psi, \theta$, and $\phi$, respectively. Each agent is attached to the load by means of a cable by means of which it can transfer forces. The cables are attached to the load at the points $L_{i}$, with $i=1, \ldots, N$, placed on the same plane, denoted by $\mathscr{I}$. The object center of mass $(\mathrm{CoM})$ is indicated with $G$.

Each cable is modeled as a linear unidirectional spring, with a dissipative term that damps its longitudinal oscillations. We assume that each flying agent is endowed with a position controller. If the latter is sufficiently precise, we can model the closed loop system as a simple double integrator. In this way we can consider each agent as an actuated point mass capable of exchanging a force with the external world.

By doing so, the proposed method can be applied to different aerial robots. If we consider multidirectional-thrust platforms capable of controlling position and orientation independently (popular in the field of aerial physical interaction) $[4,15,16,18]$, the double integrator is an exact model of the position-controlled closed loop system. In the case of underactuated unidirectional-thrust vehicles using a standard position controller, the double integrator is instead a very good approximation. Furthermore, the time-scale

Preprint version, 11th Swarm Intelligence ANTS 2018, Budapest, Hungary -- page 3/13 
separation between the translational and rotational dynamics has been exploited in other works on aerial manipulation like $[12,14]$ where the robots are considered as point masses and modeled as double integrators. Denoting by the vector $\boldsymbol{p}_{R i} \in \mathbb{R}^{3}$ the position of the $i$-th agent, by $\boldsymbol{M}_{i}=m_{A i} \boldsymbol{I}_{3}$ its inertia matrix (with $m_{A i} \in \mathbb{R}_{>0}$ ) and by $\boldsymbol{f}_{i}=\left[\begin{array}{lll}f_{i, x} & f_{i, y} & f_{i, z}\end{array}\right]^{\top}$ the force that the $i$-th cable exerts on the object (so that $-\boldsymbol{f}_{i}$ is the force exerted by the cable on the agent), the dynamics of the $i$-th agent is:

$$
\begin{aligned}
\boldsymbol{M}_{i} \ddot{p}_{R i} & =-\boldsymbol{f}_{i}+\boldsymbol{f}_{C i}, \\
\text { where } \quad \boldsymbol{f}_{C i} & =\boldsymbol{B}_{i}\left(\dot{\boldsymbol{p}}_{R i}^{d}-\dot{\boldsymbol{p}}_{R i}\right)+\boldsymbol{K}_{i}\left(\boldsymbol{p}_{R i}^{d}-\boldsymbol{p}_{R i}\right)+\boldsymbol{\pi}_{i}
\end{aligned}
$$

is the 'control' force of each agent. To better understand how to control a multi-rotor aerial robot in such a way, the reader can refer to [21]. Such control force models three simple actions. First, the agent implements a spring like action to move towards a desired position or follow a desired path (the apex ${ }^{d}$ indicates the 'desired' quantities). Secondly, the agent implements a dissipative derivative term proportional to the velocity error. This action damps the oscillations induced by the spring action. Finally, there is a force bias indicated with $\pi_{i}=\left[\pi_{i, x} \pi_{i, y} \pi_{i, z}\right]^{\top}$. This bias is essential to make the flying agents sustain the weight of the load. We shall show that it plays an important role also for shaping the system equilibria. Through $\pi_{i}$ it is possible to set reference internal forces (forces that do not result in a motion of the object).

The static equilibrium equation of the object subject to the gravity vector $g \in \mathbb{R}^{3}$ is given by $\left[\begin{array}{ll}m_{L} \boldsymbol{g}^{\top} & 0_{1 \times 3}\end{array}\right]^{\top}=W \overline{\boldsymbol{f}}$, where $W \in \mathbb{R}^{6 \times 3 N}$ is the grasp matrix, that maps the forces at the contact points to a wrench applied at the object center of mass and $\bar{f} \in$ $\mathbb{R}^{3 N \times 1}$ collects the equilibrium cable forces. Resolving the equation for $\bar{f}$ we obtain:

$$
\overline{\boldsymbol{f}}=W^{\dagger}\left[\begin{array}{ll}
m_{L} \boldsymbol{g}^{\top} & 0_{1 \times 3}
\end{array}\right]^{\top}+\boldsymbol{t}
$$

where ${ }^{\dagger}$ indicates a right (pseudo)inverse, and $t \in \operatorname{null}(W)$ is the internal force, which neither influences the object dynamics nor balances any external wrench.

Generally, in leader follower approaches it may occur that only one agent, i.e., the leader, is aware of the desired trajectory $\left(\boldsymbol{p}_{R i}^{d}, \dot{\boldsymbol{p}}_{R i}^{d}\right)$. On the other hand, slave agents tend to stay where they are if no external action intervenes. We model such agents by setting $\boldsymbol{K}_{i}=\mathbf{0} \mathrm{Nm}^{-1}$ and $\dot{\boldsymbol{p}}_{R i}^{d}=\mathbf{0} \mathrm{m} \mathrm{s}^{-1}$ in (2). When the leader starts moving, the followers will perceive a modification of the environment through a change in their cable force. This, in turn, will make the followers move toward the leader agent trying to bring back the force to the initial equilibrium value. It is worthy to note that, once each agent stops, possible load oscillations are damped thanks to the dissipative action modeled in (2).

\section{Numerical Study}

For a system of only two aerial agents and a beam/like load, in [21] we formally proved, using a Lyapunov-based approach, the stability or instability of all the possible equilibria and the passivity of the overall controlled system. However, it is not trivial to extend those theoretical results to $N>2$ and to a more generally shaped object. In particular, it is not straightforward to solve the so called equilibria inverse problem in [21], namely

Preprint version, 11th Swarm Intelligence ANTS 2018, Budapest, Hungary —- page 4/13 
Table 1: The different simulations setups (scenarios).

\begin{tabular}{l|l|c|c|c|c|} 
Goal of the study & Scenario & Internal Forces & CoM & Points $L_{i}$ & Unknown Parameters \\
\hline \multirow{3}{*}{ Internal force role } & $S_{a}$ & $\boldsymbol{t}=\mathbf{0}[\mathrm{N}]$ & $G \in \mathscr{I}$ & on a circle & none \\
\cline { 2 - 5 } & $S_{b}$ & $\boldsymbol{t}=\mathbf{0}[\mathrm{N}]$ & $G \notin \mathscr{I}$ & on a circle & none \\
\cline { 2 - 5 } & $S_{c}$ & $\boldsymbol{t} \neq \mathbf{0}[\mathrm{N}]$ & $G \notin \mathscr{I}$ & on a circle & none \\
\hline \hline Parametric uncertainty & $S_{d}$ & $\boldsymbol{t}=\mathbf{0}[\mathrm{N}]$ & $G \notin \mathscr{I}$ & random & $m_{L}, N$ (only bounds) \\
\hline
\end{tabular}

to find all the possible positions of the agents and forces in the cables for each stable pose of the load. The authors in [1] showed that such problem is very complex to solve even if just less than six cables of assigned length are used. Analytical answers to the problem are therefore difficult to reach. However, a numerical study of whether some of the properties discovered in [21] for the two-agent system apply also to the swarm case, where the number of follower agents may be arbitrary large and the object not only a bar, is equally interesting. Thus, in this section we extrapolate some conjectures on the expected behavior of the swarm system and validate these hypotheses through a wide numerical study.

Table 1 contains the description of the simulated conditions. The load has mass $m_{L}=5 \mathrm{~kg}$ and inertial matrix equal to $\boldsymbol{J}_{L}=\boldsymbol{I}_{3} \mathrm{~kg} \mathrm{~m}^{2}$. The leader agent, set as the agent 1 , is fed with a sufficiently smooth reference trajectory constituted by a $5^{\text {th }}$ order polynomial in 3D (rest to rest trajectory with zero acceleration at start and end points), which lasts $10 \mathrm{~s}$ and covers $2 \mathrm{~m} . \boldsymbol{M}_{1}=0.5 \boldsymbol{I}_{3} \mathrm{~kg}, \boldsymbol{M}_{i}=0.01 \boldsymbol{I}_{3(\mathrm{~N}-1)} \mathrm{kg}, \boldsymbol{B}_{1}=$ $100 \boldsymbol{I}_{3} \mathrm{Nsm}^{-1}, \boldsymbol{B}_{i}=1.5 \boldsymbol{I}_{3(N-1)} \mathrm{Nsm}^{-1}, \boldsymbol{K}_{1}=1000 \boldsymbol{I}_{3} \mathrm{Nm}^{-1}$, and $\boldsymbol{K}_{i}=0 \boldsymbol{I}_{3(\mathrm{~N}-1)} \mathrm{Nm}^{-1}$ for $i \neq 1$. When $\mathrm{N}>30$, we changed the apparent mass $\boldsymbol{M}_{i}$, and the damping $\boldsymbol{B}_{i}$ of the followers, i.e., for $i=2, \ldots, N$ reducing both by $90 \%$. This allows the leader agent to drag the system without applying too large forces, similarly to what happens in biological systems as described in Sec. 4.

Scenarios $S_{a}, S_{b}$ and $S_{c}$ refer to the cases in which the parameters of the system are perfectly known, and so is $N$. Based on what we demonstrated in [21] for the two agents system, we decided to investigate the role of the internal force on the object for the equilibria of the system. We considered the case in which the points $L_{i}$ lie on a circle centered around the projection of $G$ on $\mathscr{I}$ (see Figs. 2(a), 2(b), and 2(d)). We ran different simulations where $N$ is a random number between 2 and 100, and the initial pose of the object has a random value of yaw between $0 \mathrm{rad}$ and $\pi / 4 \mathrm{rad}$.

In the scenarios $S_{a}$ and $S_{b}$ the force bias in (2) is set to sustain the weight of the load without internal forces, namely $\pi_{i}=\left[\begin{array}{lll}0 & 0 & m_{L} g\end{array} / N\right]^{\top} \forall i=1, \ldots, N$, where $g$ is the intensity of $\boldsymbol{g}$. This implies vertical cables. Once the leader agent stops, the system converges to an equilibrium in which the final attitude of the transported object is not univocally determined but depends on the trajectory resulting from the initial condition and the leader desired trajectory. In particular, the results in the first line of plots of Fig. 3 shows a completely arbitrary final orientation of the load for the scenario $S_{a}$, and the second line of Fig. 3 an arbitrary final yaw for the scenario $S_{b}$.

In the scenario $S_{c}$ we set the force bias $\pi_{i}$ in (2) so that the system reaches an equilibrium with the cables forces applying non zero horizontal forces. More in detail, indicating with $\bar{l}_{i}$ the vector that connects the projection of $G$ on $\mathscr{I}$ to the position 


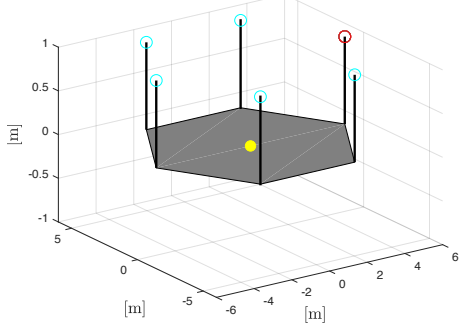

(a) $t=0$

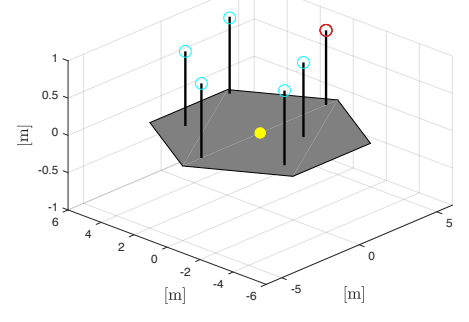

(c) cables randomly attached on the surface of the object

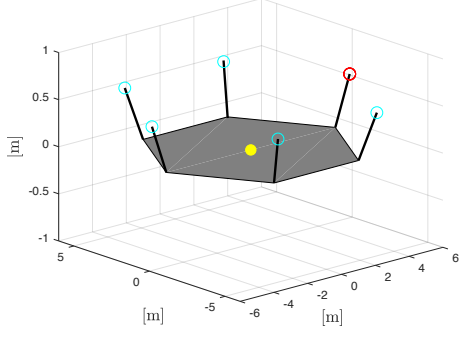

(b) $t \neq 0$

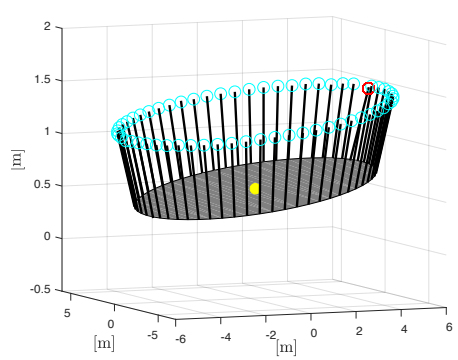

portation

Fig. 2: Simulated system for the case $N=6$ (2(a), 2(b), 2(c)) and $N=60$ (2(d))). The object is depicted as a grey surface, and the light spot on it coincide with its center of mass $G$. In case $G \notin \mathscr{I}$, the light spot is instead the projection of $G$ on $\mathscr{I}$. The cables are the black lines, while the circles represent the robots (the darker one is the leader).

of point $L_{i}$ at the final attitude, we set $\left[\pi_{i, x} \pi_{i, y}\right]^{\top}=f_{\text {int }} \bar{l}_{i} /\left\|\bar{l}_{i}\right\|$, where $f_{\text {int }}$ is thus the intensity of each agent's planar force bias. In other words, the desired horizontal forces in the cables are oriented radially and outward the object in the final configuration, similarly to what is depicted in Fig. 2(b). Since the object reaches an equilibrium, and the external wrench on the object at the equilibrium is only the vertical force due to the gravity, as in (3), such non vertical components of the cable forces, which do not cause any motion of the object and do not compensate any external wrench, generate an internal force $\boldsymbol{t} \neq \mathbf{0}$. In this way, the object reaches always the same attitude: zero pitch, zero roll, and the same yaw. The third line of Fig 3 shows the results of such simulations. Notice that the same results have been obtained even when $G \in \mathscr{I}$. Fig. 4 shows the evolution of the forces that the agents apply to the load in four different cases belonging to scenario $S_{c}$.

We also decided to see whether the value of $f_{\text {int }}$ influences the speed of convergence with which the swarm stabilizes the object in scenario $S_{c}$. Fig. 5 shows the evolution of the load attitude when transported by a group of five agents for different values of $f_{\text {int }}$. 

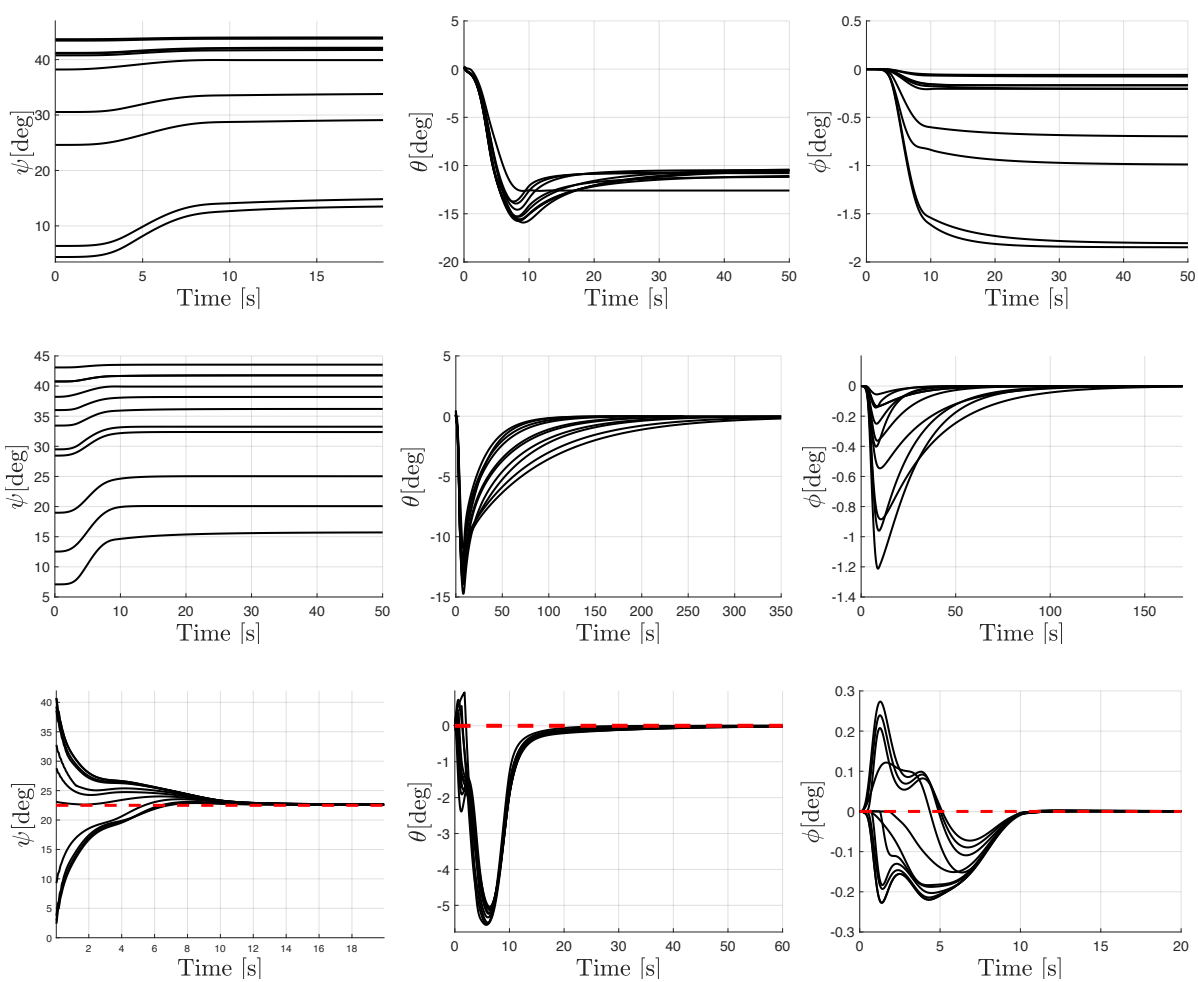

Fig. 3: Object attitude during transportation. First row: scenario $S_{a}: f_{\text {int }}=0 \mathrm{~N}$ and $G \in$ $\mathscr{I}$. Second row: ??), scenario $S_{b}: f_{\text {int }}=0 \mathrm{~N}$ and $G$ below $\mathscr{I}$. Third row: ??), scenario $S_{c}: f_{\text {int }}=0.8 \mathrm{~N}$ and $G$ placed below $\mathscr{I}$. The dotted line is the desired value. The first, second, and third columns refer to the trajectories of the yaw $(\psi)$, pitch $(\theta)$, and roll $(\phi)$ angles of the object, respectively.

We present now the results concerning scenario $S_{d}$, where neither the exact real mass of the load, $m_{L}$, nor the exact total number of agents, $N$, are known by the agents. Only some upper bounds are given, indicated with $\hat{m}$ and $\hat{N}$ respectively. Therefore, we introduced a particular choice of $\boldsymbol{\pi}_{i}$.

$$
\boldsymbol{\pi}_{i}=\left[\begin{array}{c}
0 \\
0 \\
f_{i, z}+U_{i}^{z}
\end{array}\right] \text { where } U_{i}^{z}=\left\{\begin{array}{cc}
-K_{Z} & \text { if }-\left|f_{i, z}\right|<-f_{\max } \\
K_{Z} & \text { if }-\left|f_{i, z}\right|>-f_{\min } \\
0 & \text { otherwise }
\end{array}\right.
$$

Namely, we are defining a dead-zone in the sensed vertical force such that if the robots perceive a vertical force in the cable that is inside a certain range defined by $f_{\max }$ and $f_{\min }$, they ignore it; otherwise, they apply an upward or downward force trying to restore a vertical force inside the predefined range. $K_{Z}$ is a constant that determines the respon- 

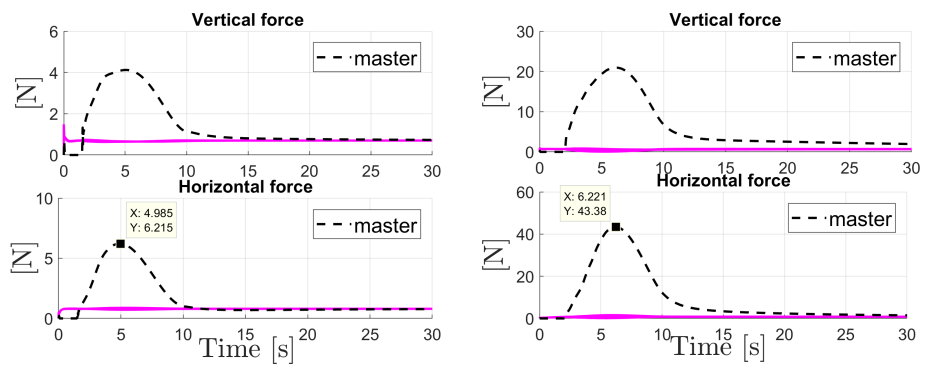

(a) $\boldsymbol{M}_{i}=0.001 \boldsymbol{I}_{3(N-1)} \mathrm{kg}$,

(b) $M_{i}=0.01 I_{3(N-1)} \mathrm{kg}$, $\boldsymbol{B}_{i}=0.15 \boldsymbol{I}_{3(N-1)} \mathrm{Nsm}^{-1}$ $\boldsymbol{B}_{i}=0.15 \boldsymbol{I}_{3(N-1)} \mathrm{Ns} \mathrm{m}^{-1}$
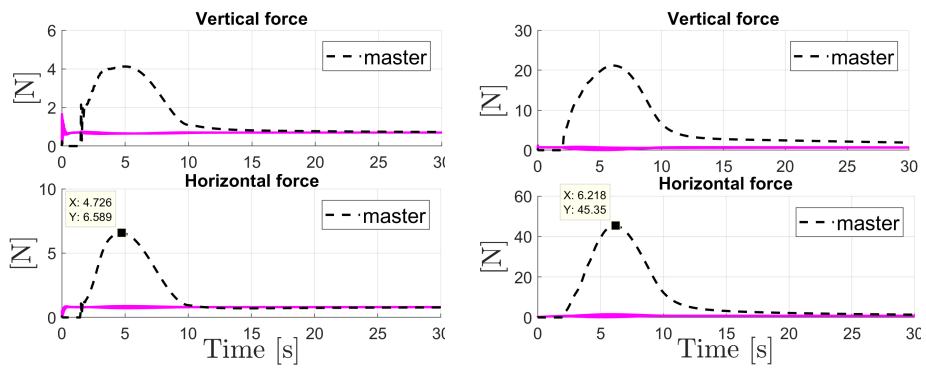
(c) $\boldsymbol{M}_{i}=0.001 \boldsymbol{I}_{3(N-1)} \mathrm{kg}$,
(d) $\boldsymbol{M}_{i}=0.01 \boldsymbol{I}_{3(N-1)} \mathrm{kg}$,
$\boldsymbol{B}_{i}=1.5 \boldsymbol{I}_{3(N-1)} \mathrm{Ns} \mathrm{m}^{-1}$
$\boldsymbol{B}_{i}=1.5 \boldsymbol{I}_{3(N-1)} \mathrm{N} \mathrm{s} \mathrm{m}^{-1}$

Fig. 4: Intensities of the vertical force $f_{i, z}$ and of the horizontal force $\sqrt{f_{i, x}^{2}+f_{i, y}^{2}}$ that the agents apply to the object during the transportation for four different parameters of the followers in scenario $S_{c}$ with $N=70$. The dotted line refers to the leader agent, while the solid lines refer to the followers. In the subcaptions $i=2, \ldots, N$.
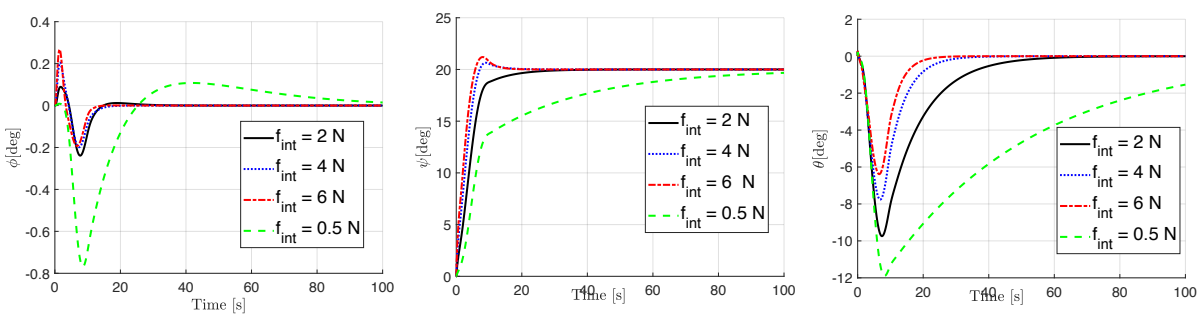

Fig. 5: Evolution of the object attitude (yaw, pitch and roll, respectively) during transportation for different values of the intensity of the internal force in scenario $S_{c}$ with $N=5$. 

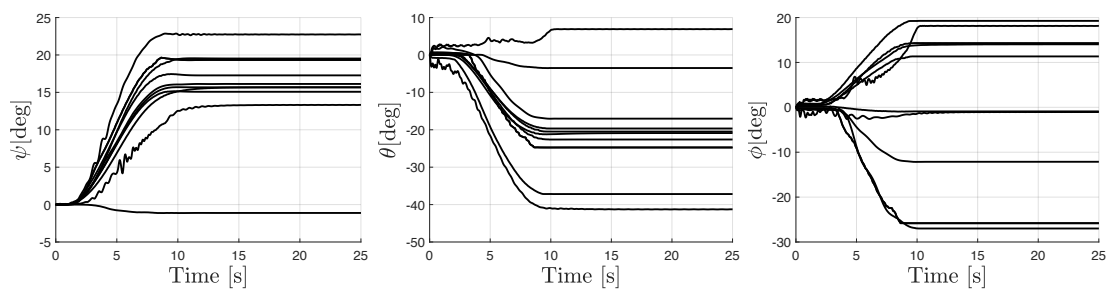

Fig. 6: Attitude of the load (yaw, pitch and roll, respectively) during the transportation task in scenario $S_{d}$ with random cables attachment points and uncertain load mass and agents number.
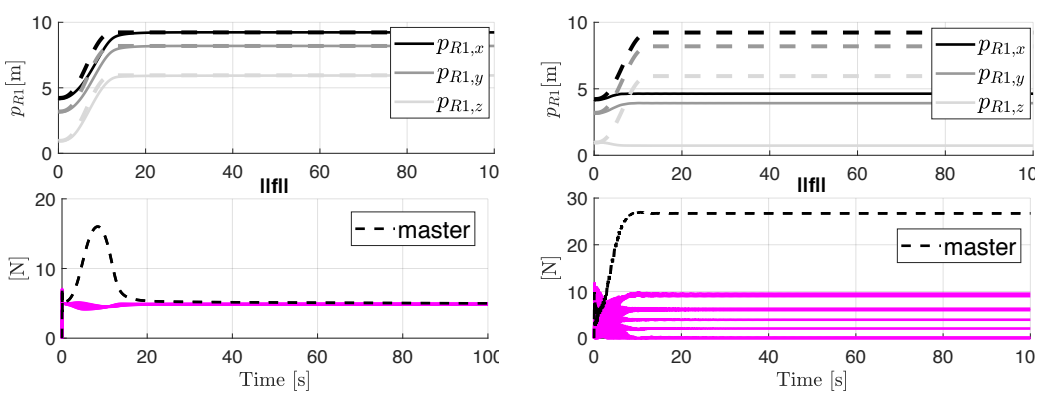

(a)

(b)

Fig. 7: The three components of $\boldsymbol{p}_{R 1}$ and $\boldsymbol{p}_{R 1}^{d}$ (dotted lines of the same color) and the intensity of the cable forces. By modeling $f_{C 1}$ as in (5) it is possible, with the same parameters, to have the leader follow its desired trajectory under normal conditions (7(a)), and to limit the forces exerted in case the swarm does not follow it (7(b)).

siveness of the robots in trying to maintain the vertical force inside $\left[-f_{\text {max }},-f_{\min }\right]$. We choose $f_{\text {max }}=\frac{\left(m_{L}+\hat{m}\right) g}{N-\hat{N}}$ and $f_{\text {min }}=\frac{\left(m_{L}-\hat{m}\right) g}{N+\hat{N}}$. In this way, we guarantee that the overall force exerted by the agents will be enough to sustain the object weight. In particular, we are not choosing a precise reference force distribution. The actual final force in each cable is induced by the choice of the bias (which does not depend on the exact values of $N$ and $m_{L}$ ) and is not the same for all the agents. Such an implementation allows the robots to successfully cope with a variation of the parameters of the swarm and of the object. Simulations results for this scenario, see Fig. 6, show that the system stabilizes after the transportation. However, due to the uncertain conditions, the final pose of the object cannot be known a priori. The number of agents varies randomly between 7 and $50, \hat{m}=0.5 \mathrm{~kg}$, and $\hat{N}=5$.

We conclude this section proposing and testing an alternative version of (2) for the leader agent. Thanks to the model (1), (2), the leader agent does not blindly follow the desired trajectory but is aware of the outer world. In the choice of the parameters 
of (2) one has to face a clear trade-off between compliance (i.e., energy consumption and safety) and tracking error (i.e., performance). A behavior that is too compliant can compromise the reach of the final position, a behavior that is too stiff can require a large amount of force from the leader side and thus a lot of energy consumption and an increased risk of ruptures. Due to space limitations we omit the performed numerical results showing such intuitive trade-off. A possible solution to better deal with such trade-off is to introduce a nonlinear saturation model like, e.g., the following one:

$$
\boldsymbol{f}_{C 1}=\boldsymbol{B}_{1} \tan ^{-1}\left(\dot{\boldsymbol{p}}_{R 1}^{d}-\dot{\boldsymbol{p}}_{R 1}\right)+\boldsymbol{K}_{i} \tan ^{-1}\left(\boldsymbol{p}_{R 1}^{d}-\boldsymbol{p}_{R 1}\right)+\boldsymbol{\pi}_{i} .
$$

The relative results are shown in Fig. 7 .

\section{Discussion}

We first simulated symmetric cables attachment points, lying on a circle around the object center of mass (or its projection on $\mathscr{I}$ ). Applying a non-zero internal force that stretches the object the agents are capable of controlling univocally the final orientation of the object without the need of communication. This result actually reflects what we had discovered and formally proven for the two-agent system and a beam-like load in [21]. It is possible to assume that also biological agents, transporting an object, may be able to pull the edge of the object towards themselves, applying a force orthogonal to the edge, and thus generating a resulting internal force altogether. For example, applying a constant horizontal force bias that equilibrates the object at the initial configuration, the swarm may be capable of transporting the load and repositioning it with the same attitude without the need of explicit communication. This suggests that the local sensing allows a sort of collective memory, as if the swarm of agents remembered the initial orientation of the object and were capable of recreating it.

We were also interested in understanding the role played by the internal forces in the convergence rate. In fact, observing some biological structures, it seems reasonable to assume that a higher internal force in the object might lead to a faster convergence of the system to the equilibrium. For example, in [2] the authors observe the dynamic response of different spider webs. It came out that an initial tension in the net changes the natural frequency in the sense that the net is faster in recovering its equilibrium after being perturbed with respect to the case without initial tension. Similarly, we found that increasing the intensity of the horizontal bias force in $\boldsymbol{f}_{C}$, and hence in turn increasing the internal force in the object, leads to faster a convergence rate, as shown in Fig. 5.

The apparent mass of the leader agent has been set to a larger value than the one of the followers. Actually, it has been observed in groups of different army ants (Eciton burchelli, Dorylus wilverthi) that the front ant in prey retrieval groups is larger than the others, and it is characterized by a larger dry weight. Additionally, in our simulations, during the dynamic part, the leader agent has to apply greater forces to the object than the other ones in the group. An example is shown in Fig. 4. However, this seems to be true also in super efficient prey retrieval groups of army ants [5], where a single submajor initiates the motion of the item by itself, and only after the rest of the group helps in the transportation. 
The damping parameter of the followers $\boldsymbol{B}_{i}$ and their masses $\boldsymbol{M}_{i}$ have been tuned depending on the number of robots. This has been done to reduce the force that the leader agent needs to apply in order to let each follower start moving. This force increases with the increasing of the damping parameters of the followers that are related to energy dissipation of the overall system. The master force in case of a small $N$ with a large damping is comparable to the force that the master applies with a large $N$ and small damping, a behavior which is reported in Fig. 4(a). To equilibrate as much as possible the force exerted by the leader for different values of $N$, one solution is to modify the damping parameter of the followers based on $N$. Another way to modify the effect of the follower inertia on the leader it is to modify their mass. Compare Figs. 4(d) and 4(b) to see the effect of the follower inertia on the master applied force, and Figs. 4(d) and 4(b) to see the effect of the damping parameter. Finally, comparing Figs. 4(d) and 4(a) the benefits of decreasing both the followers mass and damping emerges. It is not immediately clear which is the possible biological meaning of tuning the mass of the agents though. However, it is interesting to highlight that Eciton burchelli ants, very efficient in transportation tasks, tend to maintain a constant ratio between the total weight of porters and the weight of the carried load [5]. $f$ Concerning the leader spring parameter, it is not trivial to find the right balance in order to have a leader agent capable of both following the desired trajectory not being too much perturbed by small forces (for instance the ones caused by the followers initial resistance to the motion) and being sensitive to large forces on the cable indicating that something is not going as expected. However, that is exactly what an intelligent biological system would do. We were able to mimic this behavior by using a nonlinear control action on the leader agent, see (5). In this way, the leader follows the desired trajectory accurately for small deviations, but it changes its behavior consistently if the followers stay still and not follow as expected (see Fig. 7).

Finally, the results in Fig. 6, which refer to scenario $S_{d}$, show the potential of the algorithm also in conditions were the swarm is not completely aware of the parameters of the system. Even with a limited and imperfect knowledge the agents are capable of commonly carrying an object in a stable way.

\section{Conclusions}

This work is a simulative study on communication-less cooperative object transportation by swarms of aerial agents. The main focus concerns the important role of the internal force to make asymptotically stable certain equilibria of the system and to enhance the manipulation capabilities of the swarm. An imperfect knowledge of the system parameters has been also treated. Several parallelisms with biological examples are discussed as well. Validation of the proposed algorithm on real platforms and theoretical proofs is left as an important future work. Of course, in order to realize a practical implementation, additional aspects, such as a collision avoidance technique, are required, especially for very large number of agents. Furthermore, the relaxation of some hypothesis, e.g., on the cables attachment points or on the rigidity of the load, may represent an interesting enlargement of the application domain.

Preprint version, 11th Swarm Intelligence ANTS 2018, Budapest, Hungary -- page 11/13 
Acknowledgments. This research was partially supported by the ANR, Project ANR17-CE33-0007 MuRoPhen.

\section{References}

1. Abbasnejad, G., Carricato, M.: Direct geometrico-static problem of underconstrained cabledriven parallel robots with $n$ cables. IEEE Trans. on Robotics 31(2), 468-478 (2015)

2. Alam, M.S., Wahab, M.A., Jenkins, C.H.: Mechanics in naturally compliant structures. Mechanics of materials 39(2), 145-160 (2007)

3. Berman, S., Lindsey, Q., Sakar, M.S., Kumar, V., Pratt, S.C.: Experimental study and modeling of group retrieval in ants as an approach to collective transport in swarm robotic systems. Proceedings of the IEEE 99(9), 1470-1481 (2011)

4. Brescianini, D., D'Andrea, R.: Design, modeling and control of an omni-directional aerial vehicle. In: 2016 IEEE Int. Conf. on Robotics and Automation. pp. 3261-3266. Stockholm, Sweden (May 2016)

5. Franks, N.R.: Teams in social insects: group retrieval of prey by army ants (eciton burchelli, hymenoptera: Formicidae). Behavioral Ecology and Sociobiology 18(6), 425-429 (1986)

6. Franks, N.R., Sendova-Franks, A.B., Anderson, C.: Division of labour within teams of new world and old world army ants. Animal Behaviour 62(4), 635-642 (2001)

7. Gassner, M., Cieslewski, T., Scaramuzza, D.: Dynamic collaboration without communication: Vision-based cable-suspended load transport with two quadrotors. In: 2017 IEEE Int. Conf. on Robotics and Automation. pp. 5196-5202. Singapore (May 2017)

8. Grassé, P.P.: La reconstruction du nid et les coordinations interindividuelles chezbellicositermes natalensis etcubitermes sp. la théorie de la stigmergie: Essai d'interprétation du comportement des termites constructeurs. Insectes sociaux 6(1), 41-80 (1959)

9. Kube, C.R., Bonabeau, E.: Cooperative transport by ants and robots. Robotics and autonomous systems 30(1-2), 85-101 (2000)

10. Manubens Ferriol, M., Devaurs, D., Ros, G.L., Cortés, J.: A motion planning approach to 6-D manipulation with aerial towed-cable systems. In: Proceedings of the 2013 International Micro Air Vehicle Conference and Flight Competition. pp. 1-7. Toulouse, France (2013)

11. Masone, C., Bülthoff, H.H., Stegagno, P.: Cooperative transportation of a payload using quadrotors: A reconfigurable cable-driven parallel robot. In: 2016 IEEE/RSJ Int. Conf. on Intelligent Robots and Systems. pp. 1623-1630 (Oct 2016)

12. Michael, N., Fink, J., Kumar, V.: Cooperative manipulation and transportation with aerial robots. Autonomous Robots 30(1), 73-86 (2011)

13. Moffett, M.W.: Cooperative food transport by an asiatic ant. National Geographic Research 4(3), 386-394 (1988)

14. Nguyen, H.N., Park, S., Lee, D.J.: Aerial tool operation system using quadrotors as rotating thrust generators. In: 2015 IEEE/RSJ Int. Conf. on Intelligent Robots and Systems. pp. 12851291. Hamburg, Germany (Oct 2015)

15. Park, S., J. Her, J., Kim, J., Lee, D.: Design, modeling and control of omni-directional aerial robot. In: 2016 IEEE/RSJ Int. Conf. on Intelligent Robots and Systems. pp. 1570-1575. Daejeon, South Korea (2016)

16. Rajappa, S., Ryll, M., Bülthoff, H.H., Franchi, A.: Modeling, control and design optimization for a fully-actuated hexarotor aerial vehicle with tilted propellers. In: 2015 IEEE Int. Conf. on Robotics and Automation. pp. 4006-4013. Seattle, WA (May 2015)

17. Rubenstein, M., Cabrera, A., Werfel, J., Habibi, G., McLurkin, J., Nagpal, R.: Collective transport of complex objects by simple robots: theory and experiments. In: 2013 international conference on Autonomous agents and multi-agent systems. pp. 47-54 (2013)

Preprint version, 11th Swarm Intelligence ANTS 2018, Budapest, Hungary —- page 12/13 
18. Ryll, M., Muscio, G., Pierri, F., Cataldi, E., Antonelli, G., Caccavale, F., Franchi, A.: 6D physical interaction with a fully actuated aerial robot. In: 2017 IEEE Int. Conf. on Robotics and Automation. pp. 5190-5195. Singapore (May 2017)

19. Sreenath, K., Kumar, V.: Dynamics, control and planning for cooperative manipulation of payloads suspended by cables from multiple quadrotor robots. In: Robotics: Science and Systems. Berlin, Germany (June 2013)

20. Tagliabue, A., Kamel, M., Verling, S., Siegwart, R., Nieto, J.: Collaborative transportation using MAVs via passive force control. In: 2017 IEEE Int. Conf. on Robotics and Automation. pp. 5766-5773. Singapore (2016)

21. Tognon, M., Gabellieri, C., Pallottino, L., Franchi, A.: Aerial co-manipulation with cables: The role of internal force for equilibria, stability, and passivity. IEEE Robotics and Automation Letters, Special Issue on Aerial Manipulation 3(3), 2577 - 2583 (2018). https://doi.org/10.1109/LRA.2018.2803811

22. Wang, Z., Schwager, M.: Force-amplifying n-robot transport system (force-ants) for cooperative planar manipulation without communication. The International Journal of Robotics Research 35(13), 1564-1586 (2016)

23. Wu, G., Sreenath, K.: Geometric control of multiple quadrotors transporting a rigid-body load. In: 53rd IEEE Conf. on Decision and Control. pp. 6141-6148. Los Angeles, CA (Dec 2014)

Preprint version, 11th Swarm Intelligence ANTS 2018, Budapest, Hungary —- page 13/13 